\title{
The Functional Variant in the 3'UTR of IGF1 with the Risk of Gastric Cancer in a Chinese Population
}

\author{
Haiqiang Jianga,b Huiyun Wang ${ }^{\mathrm{b}}$ Fengyuan Ge ${\text { Lamei Wu } \mathrm{u}^{\mathrm{a}} \text { Xiao Wang }}^{\mathrm{a}}$ \\ Shengxia Chen ${ }^{\mathrm{a}}$
}

aDepartment of Microbiology, Jiangsu University, ZhenJiang, 'baboratory of Medicine, Chinese traditional Hospital of Jiangyin, Wuxi, China

\section{Key Words}

SNP • IGF-1 • Gastric cancer $・$ miR-603

\begin{abstract}
Background/Aims: IGF-1 can act as an endocrine hormone and its signaling server as essential roles in regulating tumorigenesis. Polymorphisms in IGF-1 have been reported associated bad prognosis of with human cancer, but their association with the risk of human gastric cancer (GC) has not been found so far. In this study rs6218 located in the 3'UTR of IGF-1 was selected to evaluate its relationship with the risk of GC among Chinese population. Methods: Questionnaire, SNaPshot genotype assay, real time PCR assay, cell transfection and the dual luciferase reporter assay were used in our study. Results: SNP rs6218 in IGF-1 3'-UTR was involved in the occurrence of GC by acting as a tumor prom0tion factor while rs6128 acting as a risk factor. SNP rs6128 was also could be regulated by miR-603 which caused an up-regulation of IGF- 1 in patients with UC and CC genotype. Furthermore, the carriers of UC and CC genotype presented a big tumor size as well as the high probability of metastasis. Conclusion: In conclusion, our findings have shown that the SNP rs6218 in IGF-1 3'-UTR, through disrupting the regulatory role of miR-603 in IGF-1 expression, rs 16128 in IGF-1 might act as a promotion factor in the pathogenesis of GC.
\end{abstract}

Copyright $@ 2015$ S. Karger AG, Basel

\section{Introduction}

Gastric cancer (GC) is defined as cancer that forms in the tissues lining the stomach. Globally, GC is the fifth leading cause of cancer and the third leading cause of cancer mortality, comprising 7\% of cases and 9\% of deaths [1]. In 2012 GC occurred in 950,000 people 
and caused 723,000 deaths [1]. The most common cause is infection by H. pylori, which accounts for $>60 \%$ of cases $[2,3]$. GC is the most common subtype with a poor treatment and survive rate $[4,5]$. Age, diet, genetic background and environmental factors are thought to be associated with risk of GC $[6,7]$. Although many people are exposed to these risk factors, only a few individuals develop GC in their lifetimes, suggesting that genetic variation may contribute to gastric cancer. Traditional treatment on GC is not enough comparing with the increasing number of people suffering with GC $[8,9]$. Improving results with the candidate gene approach have led to its growing acceptance as a potentially useful method for investigating genetic risk factors for GC among Chinese.

IGF-1 is produced primarily by the liver as an endocrine hormone as well as in target tissues via paracrine/autocrine. The IGF signaling pathway has a pathogenic role in cancer $[10,11]$. Mainly due to that the IGF1 signals triggered through the insulin receptors (IRs) and IGF1 receptor (IGF1R), respectively, result in activation of the phosphotidylinositol 3-kinase/Akt signaling pathway and protein kinase C [12]. The potential anti-tumor effect of statins has been reported for multiple IGF-1-dependent malignancies. The strong inverse correlation between stat in use and colorectal cancer, hepatocellular carcinoma, and gastric cancer [13-16].

MiRNAs are small, non-coding RNA molecules of 19-25 nucleotides which have been reported to play important roles by regulating cell differentiation, proliferation, migration and apoptosis [17]. MiRNAs can not only negatively regulate their target genes expression at the posttranscription level through binding to 3' untranslated regions (UTRs) of their targets message RNAs, $[18,19]$ but also regulate the 3'UTR region who harboured the singlenucleotide polymorphisms (SNPs). SNP is the most common human genetic variations, have been proved to be significantly related to the occurrence of diseases including gastric cancer [20]. More and more studies have provided evidences that SNPs located in the miRNA (miRSNPs) binding sites through affecting the binding of miRNAs with the target genes resulted in reduction or increase in the target mRNA translation, and thus being associated with the susceptibility to cancers [21,22].

In this study, we focused on the SNPs in the 3'UTR of IGF-1 which has rarely been reported before. By using the bioinformatics software (http://www.bioguo.org/miRNASNP/), we obtained all the SNPs which could regulate by different miRNAs as candidate SNPs and further investigated the allele distribution in a case-control study.

\section{Materials and Methods}

\section{Study subjects}

The hospital-based case-control study consists of 580 patients newly diagnosed with GC and 673 cancer-free controls. All the subjects were genetically unrelated Han Chinese recruited from the Chinese Traditional medicine hospital of Jiangyin (Nanjing, China), January 2010 and September 2014. Patients with other hematological disorders, previous history of cancers, radiotherapy and chemotherapy were excluded. The cancer-free control subjects from the same geographic area showed no evidence of genetic relationship with the cases. The patients were classified according to World Health Organization classification. This study was approved by the Ethical Committee of the Chinese Traditional medicine hospital of Jiangyin, and every patient had written informed consent.

\section{Genotype}

We extracted genomic DNA from peripheral whole blood of every validation subject by using QIAamp DNA blood mini kits (Qiagen, Germany) according to the manufacturer's instructions. Genotyping was performed with the TaqMan SNP Genotyping Assay. The PCR reactions were carried out in a total volume of 5 mL containing TaqMan Universal Master Mix, 80X SNP Genotyping AssayMix, Dnase-free water and 10-ng genomic DNA. The PCR conditions were $2 \mathrm{~min}$ at 50uC, $10 \mathrm{~min}$ at $95 \mathrm{uC}$, followed by 40 cycles at 95 for 15 sec and 60uC for $1 \mathrm{~min}$. The 384-well ABI 7900HT Real Time PCR System 


\section{Cellular Physiology Cell Physiol Biochem 2015;36:884-892 \begin{tabular}{l|l} 
and Biochemistry Publisned onIIne: June 09, 2015 & $\begin{array}{l}\text { C 2015 S. Karger AG, Basel } \\
\text { www.karger.com/cpb }\end{array}$ \\
\hline
\end{tabular} \\ Jiang et al.: SNPs in IGF1 with GC}

Real time PCR assay

Real time polymerase chain reaction (RT-PCR) was performed to determine whether the $\mathrm{C}$ to $\mathrm{T}$ mutation changed the expression level of IGF-1. The amplification conditions were $95^{\circ} \mathrm{C}$ for 10 minutes, followed by 40 cycles of $95^{\circ} \mathrm{C}$ for 30 seconds, $55^{\circ} \mathrm{C}$ for 40 seconds, and $72^{\circ} \mathrm{C}$ for 30 seconds, and finally $4^{\circ} \mathrm{C}$ for 30 minutes for cooling.

Cell lines and cell culture

GC cell lines and SGC-7901 and MKN-45 were purchased from the Chinese Academy of Sciences Cell Bank. All cells were cultured in RPMI-1640 (Gibco, USA) supplemented with $10 \%$ fetal bovine serum (Invitrogen, Carlsbad, USA) and grown in humidified $5 \% \mathrm{CO}_{2}$ at $37^{\circ} \mathrm{C}$. MiR-603 mimics and normal control were obtained from Genepharma (Shanghai, China). The transfection was conducted by using Lipofectamine 2000 (Invitrogen Corp, CA, USA).

Prediction of miRNAs binding to the SNP

Based on our bioinformatics analysis by using the bioinformatics software (http://www.bioguo.org/ miRNASNP/) to predict the related SNPs in the 3'UTR of IGF-1 which could regulated by different miRNAs.

Construction of luciferase-based reporter plasmids

All the fragment of the 3'UTR containing either $\mathrm{C}$ or T allele was amplified. The PCR production was cloned into the pGL3-promoterless luciferase-based plasmid (Promega) at the cloning site between KpnI and XhoI. The amplified fragment was verified by DNA sequencing.

Dual-luciferase reporter assay

The 3'-UTR sequence of IGF-1 predicted to interact with miR-603 or a mutated sequence with the predicted target sites were inserted into the KpnI and XhoI sites of pGL3 promoter vector (Genscript, Nanjing, China). For reporter assay, cells were plated onto 24-well plates and transfected with 100 ng of pGL3-IGF-1 wild, pGL3-IGF-1 mutant and miR-603 mimics, respectively by using Lipofectamine 2000 (Invitrogen Corp, CA, USA). A Renilla luciferase vector pRL-SV40 (5 ng) was also co-transfected to normalize the differences in transfection efficiency. Transfection was repeated three times in triplicate.

\section{Statistical analysis}

The association between rs5742714 and rs6218 genotypes and the risk of GC was evaluated by calculating the odds ratios (ORs) and their 95\% confidence intervals (CIs) using univariate and multivariate logistic regression analysis. Stratification analysis was performed according to the clinical characteristic and risk classification to determine the genotype distribution in cases and controls as well as their association with the risk of GC. The difference of the expression levels of IGF-1 with three genotypes and the difference of the relative luciferase activities between the wild and mutant genotype were evaluated by independentsample $t$ test. All statistical tests were two-sided and $\mathrm{P}<0.05$ was considered statistically significant. Statistical analysis was performed with SPSS 13.0 (SPSS Ltd.) and SAS software (version 9.1.3; SAS Institute, Cary, NC, USA). The graphs were generated by Graphpad Prism 5.0 (Graphpad Software, Inc.).

\section{Results}

\section{Subject characteristics}

The characteristics of the 570 GC patients and 673 healthy controls are summarized. No statistically significant differences were observed between cases and controls in terms of sex and age (both $\mathrm{P}>0.05$ ). This indicates that the frequency matching was adequate. Patients suffering from smoking exposure indicated to be the susceptible population by comparing with controls (Table 1).

The miRSNPs in the IGF-1 gene 3'-UTR

In order to investigate the miRNA associated SNPs in the 3'UTR of IGF-1, we first found all the SNPs from the SNP databases NCBI db SNP BUILED 129 and ENSEMBL v58 in the 
Jiang et al.: SNPs in IGF1 with GC

3'-UTR of IGF-1 gene with the minor allele frequency $(\mathrm{MAF})>0.05$. We then used bioinformatic softwares Diana-Micro, MicroInspector, miR-anda, miRNAMap, RNAhybrid to predict miRNAs that can bind to the IGF-1 3'-UTR. The miRNASNP database was also applied to explore the miRNAs which could also binding to the 3'-UTR of patients harbored the SNP. As shown in Table 2. Finally, we obtained 2 SNPs in the 3'UTR which could be regulated by three different miRNAs (miR-603, miR-580 and miR-3941). The positions of the SNPs in IGF-1 as well as the variants were listed. Further genotyping was performed to detect the distribution of allele gene of the 3 SNPs in our research.

Correlation of rs5742714 and rs6218 with gastric cancer

Interestingly, two SNPs (rs5742714 and rs6218) indicated a significant difference in GC patients respectively; as listed in Table 3, Chi-square statistical analysis results showed that the genotypes of rs5742714 were in Hardy-Weinberg equilibrium distribution pattern in the healthy control group $(\mathrm{P}=0.0092)$. Further, logistic regression analysis results revealed that The GC genotype and CC genotype presented a significant decreased risk of GC as compared with the GG genotype. The IGF1 rs6128 $\mathrm{U}$ allele was also shown to be a risk allele $(\mathrm{P}=0.0041)$. Logistic regression analyses indicated that individuals with the rs6128 AC genoty-
Table 1. Frequency distributions of selected variables in patients and cancer-free controls. *Two-sided chi-square test for either genotype distributions or allele frequencies between cases and controls

\begin{tabular}{|c|c|c|c|c|c|}
\hline \multirow{2}{*}{ Variables } & \multicolumn{2}{|c|}{ Cases $(n=570)$} & \multicolumn{2}{|c|}{ Controls $(n=673)$} & \multirow{2}{*}{$P$} \\
\hline & $N$ & $\%$ & $N$ & $\%$ & \\
\hline Age (years) & & & & & 0.254 \\
\hline$\leq 60$ & 313 & 54.91 & 348 & 51.71 & \\
\hline$>60$ & 257 & 45.09 & 325 & 48.29 & \\
\hline Gender & & & & & 0.354 \\
\hline Male & 258 & 45.26 & 287 & 42.64 & \\
\hline Female & 312 & 54.74 & 386 & 57.36 & \\
\hline Parental smoking status & & & & & 0.073 \\
\hline Negative & 278 & 48.77 & 294 & 43.68 & \\
\hline Positive & 292 & 51.23 & 379 & 56.32 & \\
\hline \multicolumn{6}{|l|}{ Differentiation grade } \\
\hline Well & 263 & 52.81 & & & \\
\hline Moderate & 229 & 47.19 & & & \\
\hline Poorly & 78 & & & & \\
\hline \multicolumn{6}{|l|}{ Tumor Size $(\mathrm{cm})$} \\
\hline$\leq 3 \mathrm{~cm}$ & 286 & 50.18 & & & \\
\hline$>3 \mathrm{~cm}$ & 284 & 49.82 & & & \\
\hline \multicolumn{6}{|l|}{ Tumor Number } \\
\hline Solitary & 316 & 55.44 & & & \\
\hline Multiple & 254 & 44.56 & & & \\
\hline \multicolumn{6}{|l|}{ Metastasis } \\
\hline Yes & 306 & 53.68 & & & \\
\hline No & 264 & 46.32 & & & \\
\hline
\end{tabular}

Table 2. Association between SNPs and Gastric cancer. $\mathrm{Chr}=$ chromosome; UTR= Untranslated Regions

\begin{tabular}{ccccc}
\hline SNP & chr & $\begin{array}{c}\text { 3'UTR } \\
\text { position }\end{array}$ & $\begin{array}{c}\text { Associated } \\
\text { miRNA }\end{array}$ & Allele* \\
\hline rs5742714 & 12 & $6416-6435$ & miR-580 & G/C \\
rs6218 & 12 & $2636-2657$ & miR-603 & U/C \\
rs6218 & 12 & $2640-2658$ & miR-3941 & U/C \\
\hline
\end{tabular}

Table 3. Genotype frequencies of the IGF-1 rs574271 and rs6218 polymorphism among GC cases and controls. ${ }^{\text {aThe }}$ ORs, 95\% CIs and $P$ value were calculated after adjusting for age, gender, parental smoking, drinking and family caner history

\begin{tabular}{|c|c|c|c|c|c|c|}
\hline \multirow{2}{*}{ Genotype } & \multicolumn{2}{|c|}{ Cases $(n=570)$} & \multicolumn{2}{|c|}{ Controls $(n=673)$} & \multirow{2}{*}{$\begin{array}{c}\text { OR } \\
(95 \% \mathrm{CI})^{\mathrm{a}}\end{array}$} & \multirow{2}{*}{$P$ Value } \\
\hline & $N$ & $\%$ & $N$ & $\%$ & & \\
\hline \multicolumn{7}{|c|}{ rs5742714 } \\
\hline GG & 328 & 57.54 & 368 & 54.68 & 1.00 & 0.0092 \\
\hline $\mathrm{GC}$ & 177 & 31.05 & 253 & 37.59 & $1.04(1.34-1.93)$ & \\
\hline $\mathrm{CC}$ & 75 & 13.16 & 52 & 7.73 & $1.802(1.27-1.79)$ & \\
\hline \multicolumn{7}{|l|}{ rs6218 } \\
\hline UU & 286 & 50.18 & 376 & 55.87 & 1.00 & 0.0041 \\
\hline UC & 219 & 38.42 & 245 & 36.40 & $1.18(1.45-1.79)$ & \\
\hline $\mathrm{CC}$ & 75 & 13.16 & 52 & 7.73 & $1.90(1.36-1.79)$ & \\
\hline
\end{tabular}




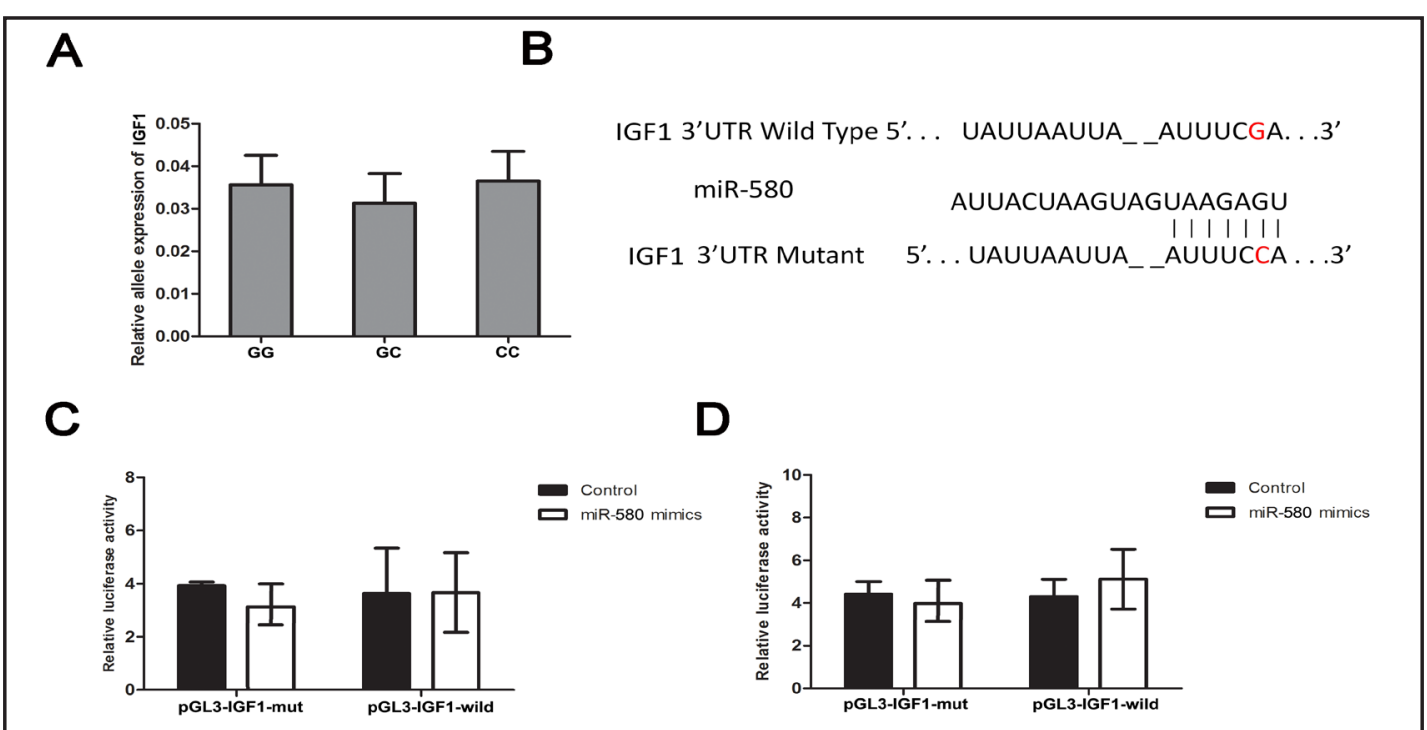

Fig. 1. miR-508 could not regulate 3'-UTR of IGF-1 of patients with GC/CC genotypes of SNP rs5742714. A: The expression level of IGF-1 was determined by RT-PCR in patients with GG, GC and CC genotypes. B: Bioinformatics predicted the binding site between the miR-508 with IGF-1 and the mutation types were conducted into the pGL3 plasmid as presented. C: Cells were co-transfected with miR-508 mimics or control, Renilla luciferase vector pRL-SV40 for $48 \mathrm{~h}$. Both firefly and Renilla luciferase activities were measured in the same sample. Firefly luciferase signals were normalized with Renilla luciferase signals. Left panel indicated the SGC-7901 cell line while the right indicated MNK45 cell lines. Data was presented as the mean \pm SEM. * indicates a significant difference $(\mathrm{P}<0.05)$.

pe was significantly associated with gastric cancer risk (OR=1.18, 95\% CI=1.45-1.79). Individuals having the rs6128 CC genotype had an OR of 1.90 (95 \% CI=1.36-1.79) for GC compared with individuals having the rs6128 CC genotype. All ORs were adjusted for sex, age, and smoking status, drinking history or family cancer history.

The effect of rs5742714 and rs6128 on the regulatory role of miRNAs in IGF-1 expression

Since the SNP rs5742714 and rs6128 were predicted to locate in the binding site of miR580 and miR-603 or miR-3941, respectively. We hypothesized that the expression of IGF-1 might be regulated by these microRNAs, which can be impacted by rs5742714 and rs6128. To test whether or not the inhibitory role of these miRNAs impacted by the two SNPs, we first detected the expression level of IGF-1 expression level in patients harbored the GG, GC and CC genotypes as well as the patients with UU, UC and CC genotypes. We found that patients with UC or CC genotypes presented a significantly increased level of IGF-1 by comparing with the patients with UU genotype; however there was no difference in rs5742714 which has GG, GC and CC genotypes (Fig. 1A, 2A). We then constructed pGL3 vectors including the allele-specific binding sequences (Fig. 1B, 2B and 3A), and then co-transfected it with miR508, miR-603 or miR-3941 as well as the controls in GC cell lines including SGC-7901 and MKN45. As presented in Fig. 1C and 1D, we found that the expression of C-allele-specific of both pGL3 construct was not significantly suppressed by miR-580 on rs5742714 as well as C allele-specific mutation on rs6128 by miR-3941 (Fig. 1C,D and Fig. 3B,C) However, the IGF-1 promoter activity were significantly suppressed by miR-603 by C-allele-specific mutation on rs6128. These findings suggested that the inhibitory affection of the UC or CC genotype of IGF-1 might be regulated by miR-603.

Stratified analyses of association between IGF-1 polymorphism and GC risk

Then, we did stratified analysis of the association of the rs6218 genotypes with the clinicopathological parameters of GC (Table 4). We found a significant association of the 


\section{A \\ B}

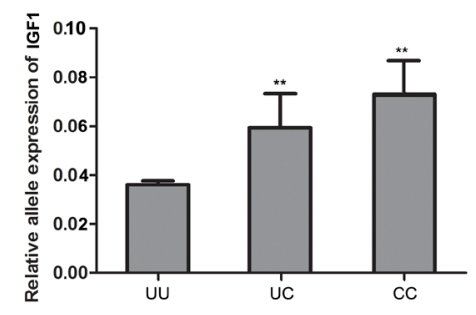

IGF1 3'UTR Wild Type 5'. . . UAUUUCUUGUCCCCAGUGUGUA. . .3' miR-603

IGF1 3'UTR Mutant $\quad 5^{\prime}$... UAUUUCUUGUCCCCAGCGUGUA. . .3'

C

D
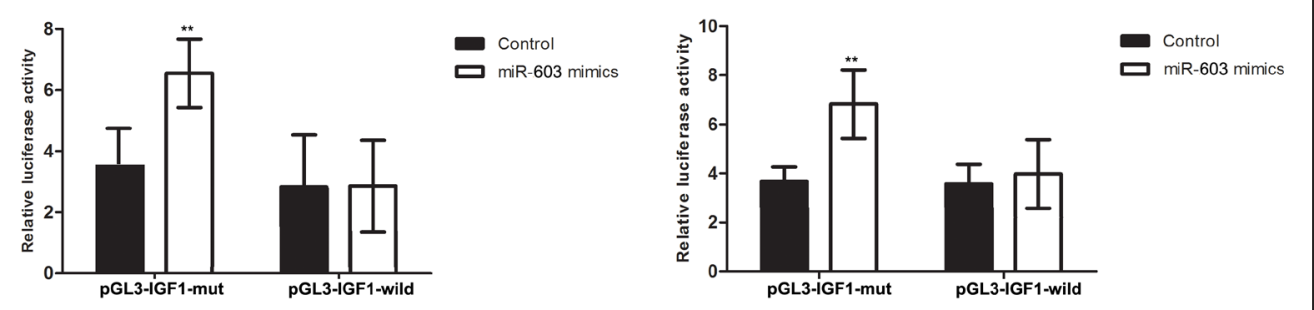

Fig. 2. SNP rs6218 in 3'UTR of GC patients with UC/CC genotype can up-regulate of IGF-1 transcription by deregulated by miR-603. A: The expression level of IGF-1 was determined by RT-PCR in patients with UU, UC and CC genotypes. B: Bioinformatics predicted the binding site between the miR-603 with IGF-1 and the mutation types were conducted into the pGL3 plasmid as presented. C: Cells were co-transfected with miR-603 mimics or control, Renilla luciferase vector pRL-SV40 for $48 \mathrm{~h}$. Both firefly and Renilla luciferase activities were measured in the same sample. Firefly luciferase signals were normalized with Renilla luciferase signals. Left panel indicated the SGC-7901 cell line while the right indicated MNK45 cell lines. Data was presented as the mean \pm SEM. * indicates a significant difference $(\mathrm{P}<0.05)$.

\section{A}

IGF1 3'UTR Wild Type 5'... U UCUUGUCCCCA----GUGUGUA. . .3'

miR-3941

ACUAGGAGUCAAACACACAUU

IGF1 3’UTR Wild Type 5'... I UCUUGUCCCCA---GCGUGUAC. . .3'

B<smiles>[CH3-]</smiles>
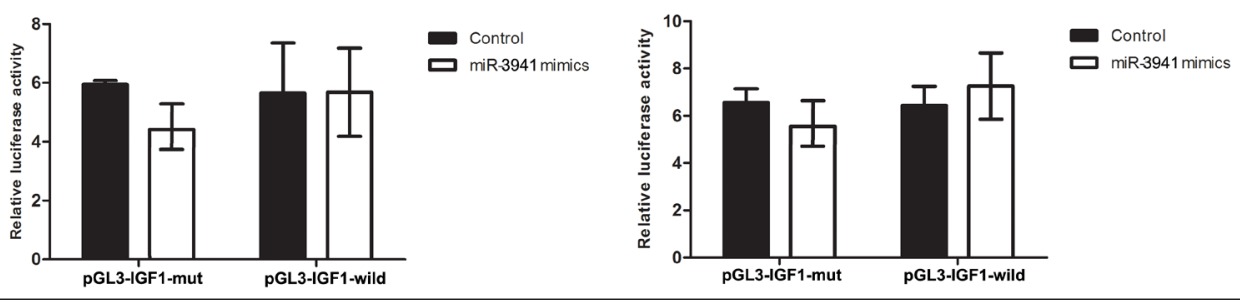

Fig. 3. miR-3941 could not regulate 3'-UTR of IGF-1 of patients with UC/CC genotypes of SNP rs6218. A: Bioinformatics predicted the binding site between the miR-3941 with IGF-1 and the mutation types were conducted into the pGL3 plasmid as presented. C: Cells were co-transfected with miR-3941 mimics or control, Renilla luciferase vector pRL-SV40 for $48 \mathrm{~h}$. Both firefly and Renilla luciferase activities were measured in the same sample. Firefly luciferase signals were normalized with Renilla luciferase signals. Left panel indicated the SGC-7901 cell line while the right indicated MNK45 cell lines. Data was presented as the mean \pm SEM. * indicates a significant difference $(\mathrm{P}<0.05)$. 


\begin{tabular}{|c|c|c|}
\hline Cellular Physiology & Cell Physiol Biochem 2015;36:884-892 & \\
\hline and Biochemistry & $\begin{array}{l}\text { DOI: 10.1159/000430263 } \\
\text { Publisnea onine:June v9, } 2015\end{array}$ & $\begin{array}{l}\text { O } 2015 \mathrm{~S} \text {. Karger AG, Basel } \\
\text { www.karger.com/cpb }\end{array}$ \\
\hline
\end{tabular}

rs6128 genotypes with the tumor size, and tumor metastasis. Compared with the UU homozygote, the carriers of UC and CC genotype presented a small tumor size as well as the low probability of metastasis.

\section{Discussion}

In the present study, we investigated the relationship between miRSNPs within the 3'-UTR of IGF-1 gene and the risk of GC. We observed that IGF-1 rs2866943 CT or TT genotype is associated with significantly decreased GC risk compared with CC genotype and the increased risk of $\mathrm{CC}$ and AC genotypes comparing with AA genotype of SNP rs6029959. We further found that the SNP rs2866943, locating in the binding sites of miR-218 through disrupting the inhibitory role of miR-218 on IGF-1 expression, played an important role in the development of GC.

IGF1 was found to be SNP and related to clinical features of many malignancies including prostate cancer, breast cancer,
Table 4. Stratified analysis of rs6218 genotype with clinicopathological parameters of GC *Two-sided chi-square test for either genotype distributions or allele frequencies between cases and controls

\begin{tabular}{lccccc}
\hline Feather & & & & $\begin{array}{c}\text { UU vs UC } \\
P \text { Value }\end{array}$ & $\begin{array}{c}\text { UU vs CC } \\
P \text { Value }\end{array}$ \\
\hline Age (years) & UU & UC & CC & (c) \\
$\leq 60$ & 108 & 115 & 35 & 0.94 & 0.24 \\
$>60$ & 124 & 134 & 54 & & \\
Gender & & & & & \\
Male & 148 & 109 & 56 & 0.22 & 0.31 \\
Female & 116 & 107 & 34 & & \\
Differentiation grade & & & & & \\
Well & 95 & 107 & 61 & 0.33 & $<0.0001$ \\
Moderate & 108 & 87 & 34 & & \\
Poorly & 42 & 33 & 3 & & \\
Tumor Size(cm) & & & & & \\
$\leq 3$ cm & 157 & 99 & 30 & $<0.0001$ & $<0.0001$ \\
$>3$ cm & 80 & 142 & 62 & & \\
Tumor Number & & & & & \\
Solitary & 124 & 109 & 83 & 0.28 & 0.06 \\
Multiple & 118 & 84 & 52 & & \\
Metastasis & & & & & \\
Yes & 137 & 124 & 80 & 0.28 & $<0.0001$ \\
No & 87 & 97 & 45 & & \\
\hline
\end{tabular}

cervical cancer, etc. Early in 2007, variation in the 5 '-untranslated region (UTR) of the IGF-1 and IGFBP-3 genes may be influencing IGF serum levels and prostate cancer risk in AfricanAmericans [23]. SNP C $>\mathrm{T}$ at the -383 position of P1 promoter may be one of the helpful prognostic markers in the diagnosis of cervical cancer development of women with persistent infection in the ectocervical epithelium. And IGF1 rs2946834 polymorphism is associated with clinical outcome of HER2-positive breast cancer patients. However, no snp reports can be found about gene polymorphisms of IGF-1 was related to gastric cancer, however, the overexpression of IGF-1 was found to be seriously related to carcinogenesis and metastasis of gastric cancer $[13,14,24,25]$. We investigated that there is two snp locis in the 3'UTR region of IGF1 and could potentially bind to three miRNAs, and after further investigation, the mutation of rs6218 can effectively affect the binding of miR-603 to 3'UTR region of IGF-1 can affect the activity of promoter of IGF- 1 can further increase the transcription of IGF-1, which can serve as one of the epigenic factors of increased production of IGF-1 in human gastric cancer.

In summary, we reported the first evidence that the SNP rs5742714 and rs6218 in IGF13 '-UTR was involved in the occurrence of GC by acting as a protective factor. SNP rs6218 was also could be regulated by miR-603 which caused a up-regulation of IGF-1 in patients with UC and CC genotype. This SNP was also found to be related to the clinicopathological features of GC, suggesting it may have important roles in promoting tumor development. Our results support the hypothesis that genetic variants interrupting miRNAs mediated regulation tumor suppressors would be involved in gastric cancer etiology.

\section{Disclosure Statement}

The authors declare that they have no financial conflict of interest. 


\section{Cellular Physiology Cell Physiol Biochem 2015;36:884-892 \begin{tabular}{l|l} 
DOI: 10.1159/000430263 & (C) 2015 S. Karger AG, Basel
\end{tabular} \\ Jiang et al:: SNPs in IGF1 with GC}

\section{References}

1 McLean MH, El-Omar EM: Genetics of gastric cancer. Nat Rev Gastroenterol Hepatol 2014;10.1038/ nrgastro.2014.143

-2 Sahin IH, Hassan MM, Garrett CR: Impact of non-steroidal anti-inflammatory drugs on gastrointestinal cancers: current state-of-the science. Cancer Lett 2014;345:249-257.

-3 Wang F, Meng W, Wang B, Qiao L: Helicobacter pylori-induced gastric inflammation and gastric cancer. Cancer Lett 2014;345:196-202.

-4 Subramanyam D, Lamouille S, Judson RL, Liu JY, Bucay N, Derynck R, Blelloch R: Multiple targets of miR302 and miR-372 promote reprogramming of human fibroblasts to induced pluripotent stem cells. Nat Biotechnol 2011;29:443-448.

5 Qin YR, Tang H, Xie F, Liu H, Zhu Y, Ai J, Chen L, Li Y, Kwong DL, Fu L, Guan XY: Characterization of tumorsuppressive function of SOX6 in human esophageal squamous cell carcinoma. Clin Cancer Res 2011;17:4655.

6 Vinci S, Gelmini S, Pratesi N, Conti S, Malentacchi F, Simi L, Pazzagli M, Orlando C: Genetic variants in miR146a, miR-149, miR-196a2, miR-499 and their influence on relative expression in lung cancers. Clin Chem Lab Med 2011;49:2073-2080.

7 Wang H, Zhang P, Chen W, Feng D, Jia Y, Xie LX: Evidence for serum miR-15a and miR-16 levels as biomarkers that distinguish sepsis from systemic inflammatory response syndrome in human subjects. Clin Chem Lab Med 2012;50:1423-1428.

8 Hirajima S, Komatsu S, Ichikawa D, Takeshita H, Konishi H, Shiozaki A, Morimura R, Tsujiura M, Nagata H, Kawaguchi T, Arita T, Kubota T, Fujiwara H, Okamoto K, Otsuji E: Clinical impact of circulating miR-18a in plasma of patients with oesophageal squamous cell carcinoma. Br J Cancer 2013;bjc2013148 [pii]. Doi 10.1038/bjc.2013.148

- Wang ZW, Zhang W, Dong W, Li BS, Mu DB, Huang W, Zhang J, Li HS, Zhang ZC, Lin HQ, Yi Y: Pathological analysis of extracapsular extension of metastatic lymph node and its potential impact on nodal clinical target volume in the radiotherapy of esophageal squamous cell carcinoma. Neoplasma 2014;61:324-330.

10 Tian Z, Yao G, Song H, Zhou Y, Geng J: IGF2R expression is associated with the chemotherapy response and prognosis of patients with advanced NSCLC. Cell Physiol Biochem 2014;34:1578-1588.

11 Chen J, Zhao X, Ye Y, Wang Y, Tian J: Estrogen receptor beta-mediated proliferative inhibition and apoptosis in human breast cancer by calycosin and formononetin. Cell Physiol Biochem 2013;32:1790-1797.

12 Brana I, Berger R, Golan T, Haluska P, Edenfield J, Fiorica J, Stephenson J, Martin LP, Westin S, Hanjani P, Jones MB, Almhanna K, Wenham RM, Sullivan DM, Dalton WS, Gunchenko A, Cheng JD, Siu LL, Gray JE: A parallel-arm phase I trial of the humanised anti-IGF-1R antibody dalotuzumab in combination with the AKT inhibitor MK-2206, the mTOR inhibitor ridaforolimus, or the NOTCH inhibitor MK-0752, in patients with advanced solid tumours. Br J Cancer 2014;111:1932-1944.

13 Gryko M, Kisluk J, Cepowicz D, Zinczuk J, Kamocki Z, Guzinska-Ustymowicz K, Pryczynicz A, Czyzewska J, Kemona A, Kedra B: Expression of insulin-like growth factor receptor type 1 correlate with lymphatic metastases in human gastric cancer. Pol J Pathol 2014;65:135-140.

14 Li S, Lei X, Zhang J, Yang H, Liu J, Xu C: Insulin-like growth factor 1 promotes growth of gastric cancer by inhibiting foxo1 nuclear retention. Tumour Biol 2015;10.1007/s13277-015-3096-9

-15 Tamura F, Sato Y, Hirakawa M, Yoshida M, Ono M, Osuga T, Okagawa Y, Uemura N, Arihara Y, Murase K, Kawano Y, Iyama S, Takada K, Hayashi T, Sato T, Miyanishi K, Kobune M, Takimoto R, Kato J: RNAi-mediated gene silencing of ST6GalNAc I suppresses the metastatic potential in gastric cancer cells. Gastric Cancer 2014;10.1007/s10120-014-0454-Z

16 Li H, Li Y, Liu D, Sun H, Liu J: miR-224 is critical for celastrol-induced inhibition of migration and invasion of hepatocellular carcinoma cells. Cell Physiol Biochem 2013;32:448-458.

17 Plasterk RH: Micro RNAs in animal development. Cell 2006;124:877-881.

18 Valastyan S, Reinhardt F, Benaich N, Calogrias D, Szasz AM, Wang ZC, Brock JE, Richardson AL, Weinberg RA: A pleiotropically acting microRNA, miR-31, inhibits breast cancer metastasis. Cell 2009;137:10321046.

19 Hyun S, Lee JH, Jin H, Nam J, Namkoong B, Lee G, Chung J, Kim VN: Conserved MicroRNA miR-8/miR-200 and its target USH/FOG2 control growth by regulating PI3K. Cell 2009;139:1096-1108. 


\section{Cellular Physiology Cell Physiol Biochem 2015;36:884-892}

and Biochemistry $\begin{gathered}\text { Dor: } 10.1159 / 000430263 \\ \text { Publisnea onine: June } 09,2015\end{gathered}$

Jiang et al.: SNPs in IGF1 with GC

-20 Wang K, Diskin SJ, Zhang H, Attiyeh EF, Winter C, Hou C, Schnepp RW, Diamond M, Bosse K, Mayes PA, Glessner J, Kim C, Frackelton E, Garris M, Wang Q, Glaberson W, Chiavacci R, Nguyen L, Jagannathan J, Saeki N, Sasaki H, Grant SF, Iolascon A, Mosse YP, Cole KA, Li H, Devoto M, McGrady PW, London WB, Capasso M, Rahman N, Hakonarson H, Maris JM: Integrative genomics identifies LMO1 as a neuroblastoma oncogene. Nature 2011;469:216-220.

21 Bartel DP: MicroRNAs: genomics, biogenesis, mechanism, and function. Cell 2004;116:281-297.

22 Wang Y, Zhou L, Chen J, Li J, He L, Wu P, Wang M, Tong N, Zhang Z, Fang Y: Association of the 3'UTR FOXO3a polymorphism rs4946936 with an increased risk of childhood acute lymphoblastic leukemia in a Chinese population. Cell Physiol Biochem 2014;34:325-332.

-23 Hernandez W, Grenade C, Santos ER, Bonilla C, Ahaghotu C, Kittles RA: IGF-1 and IGFBP-3 gene variants influence on serum levels and prostate cancer risk in African-Americans. Carcinogenesis 2007;28:21542159.

$24 \mathrm{Gu}$ MJ, Bae YK, Choi JH: Clinical significance of insulin-growth factor 1 and insulin-growth factor 1 receptor expression in gastrointestinal stromal tumors. Hepatogastroenterology 2013;60:1383-1386.

25 Li H, Xu L, Zhao L, Ma Y, Zhu Z, Liu Y, Qu X: Insulin-like growth factor-I induces epithelial to mesenchymal transition via GSK-3beta and ZEB2 in the BGC-823 gastric cancer cell line. Oncol Lett 2015;9:143-148. 